\title{
Nanosilicon Crystallite Embedded into Amorphous Silicon Matrix: Polymorphous Silicon thin film, obtained by Plasma Enhanced Chemical Vapor Deposition.
}

A. Remolina , G. Santana*, A. Ponce ${ }^{* *}$, B. M. Monroy ${ }^{*}$, M. F. García-Sánchez , J. C. Alonso ${ }^{*}$, A. Ortiz

* Instituto de Investigaciones en Materiales, Universidad Nacional Autónoma de México. A.P. 70-

360, Coyoacán 04510, México, D.F.

** Centro de Investigación en Química Aplicada, Blvd Enrique Reyna Hermosillo, 140.

Departaento de Materiales Avanzados, Saltillo, Coahuila, México.

Polymorphous silicon hydrogenated (pm-Si:H) is a nanostructured material. It is made up nanosilicon crystallite embedded in amorphous silicon matrix [1]. Currently this material is subject research worldwide, for its advantages over the standard amorphous silicon (a-Si:H). The polymorphous silicon hydrogenated provides better transport properties and response to StaeblerWronski effect than amorphous silicon standard [2].

We have obtained the polymorphous silicon ( $\mathrm{pm}-\mathrm{Si}$ : $\mathrm{H})$ from dichlorosilane $\left(\mathrm{SiH}_{2} \mathrm{Cl}_{2}\right)$, argon (Ar) and hydrogen $\left(\mathrm{H}_{2}\right)$ mixture. The plasma enhanced chemical vapor deposition (PECVD), with RFpower of $13.6 \mathrm{MHz}$ was used. The samples were grown at $200{ }^{\circ} \mathrm{C}$ in a chamber pressure at $250 \mathrm{mT}$. The dichlorosilane gas has an ease bond breakdown, using low RF power during the process of growth of the thin film. The use $\mathrm{SiH}_{2} \mathrm{Cl}_{2}$ as precursor of silicon to made up nanostructured thin film is a easy form to obtained this material. It has not been reported to obtain pm-Si:H still. The chlorine keep a balance of hydrogen into the plasma, preventing too much hydrogen enters to the film and remaining hydrogen yield crystallization into the thin film during growth process [3].

The High Resolution Transmission Electron Microscopy (HRTEM) results was obtained in a floated pm-Si:H thin film deposited on sodium chloride monocrystal. This process was taken after dilute the substrate in water and collecting it on a cooper grid used for the HRTEM analysis. In this way, we ensure that no modification of the thin film structure is produced as could be the case in conventional ion milling techniques used to prepare samples for HRTEM. The fig. 1a is a micrograph obtained by HRTEM. It shows nanocrystals silicon embedded in amorphous silicon matrix. The presence of nanocrystals into the thin film can be observed in the Fig. 1a, with a density of $8.55 \times 10^{10} / \mathrm{cm}^{2}$ of nanocrystals silicon. The amorphous structure is confirmed to bulk material by diffraction pattern as shown inset the top left of fig. 1b. The concentric rings corroborate an amorphous structure. This structure is assigned to the matrix of the thin film. The fig. 1b shows the size distribution of nanocrystals silicon embedded into the matrix. The size of nanocrystals silicon varies in a range between 4 and $14 \mathrm{~nm}$ of diameter, but most are distributed between 6 and $11 \mathrm{~nm}$. The Fig. 2a presents oval nanocrystal silicon that has $6 \mathrm{~nm}$ of diameter approximately. Its crystalline structure was verified by the Fourier Transform of the nanocrystal silicon as shown in the fig. $2 \mathrm{~b}$. The fig. $2 \mathrm{c}$ shows measurements of interplanar distance for this nanocrystal silicon. This measurements corresponding to $0.3119 \mathrm{~nm}$ to the planes (111). This result is in agreement with the theoretical distances to crystal silicon which is $0.3136 \mathrm{~nm}$ to the planes (111).

The technique of PECVD used to obtain this material is a versatile technique. The pm-Si:H is a promissory material to applications in photovoltaics devices. 


\section{References}

[1] P. Roca i Cabarroca, A. Fontcuberta i Moral, Y. Poissant. Thin Solid Film 403 (2002) 39.

[2] D. E. Carlson, C. R. Wronski. Appl. Phys. Lett. 28 (1976) 671.

[3] G. Santana, B. M. Monroy, A. Ortiz, L. Huerta, and J. C. Alonso. Appl. Phys. Lett. 88 (2006) 041916.

[4] We acknowledge partial financial support for this work from CONACyT México, under projects $47303-\mathrm{F}$ and 48970 and A. Remolina Millán thanks for the scholarship grant by Instituto de Ciencia y Tecnología del Distrito Federal (ICyTDF), Mexico D.F.
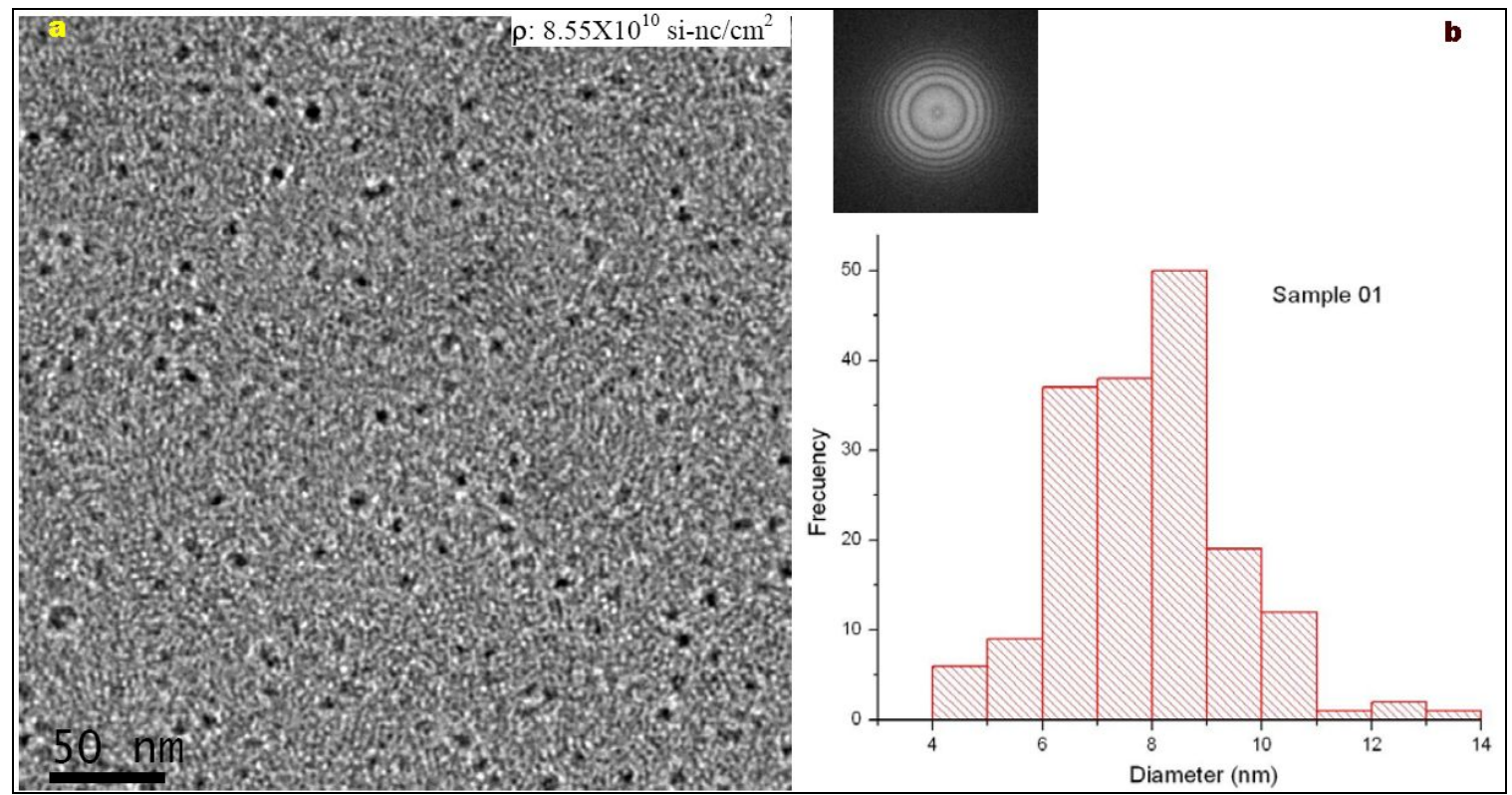

Fig. 1. Polymorphous silicon hydrogenated (pm-Si:H). a) Nanocrystal embedded into amorphous matrix. b) Inset right upper, pattern diffraction of matrix. Figure down size nanocrystal distribution into matrix.

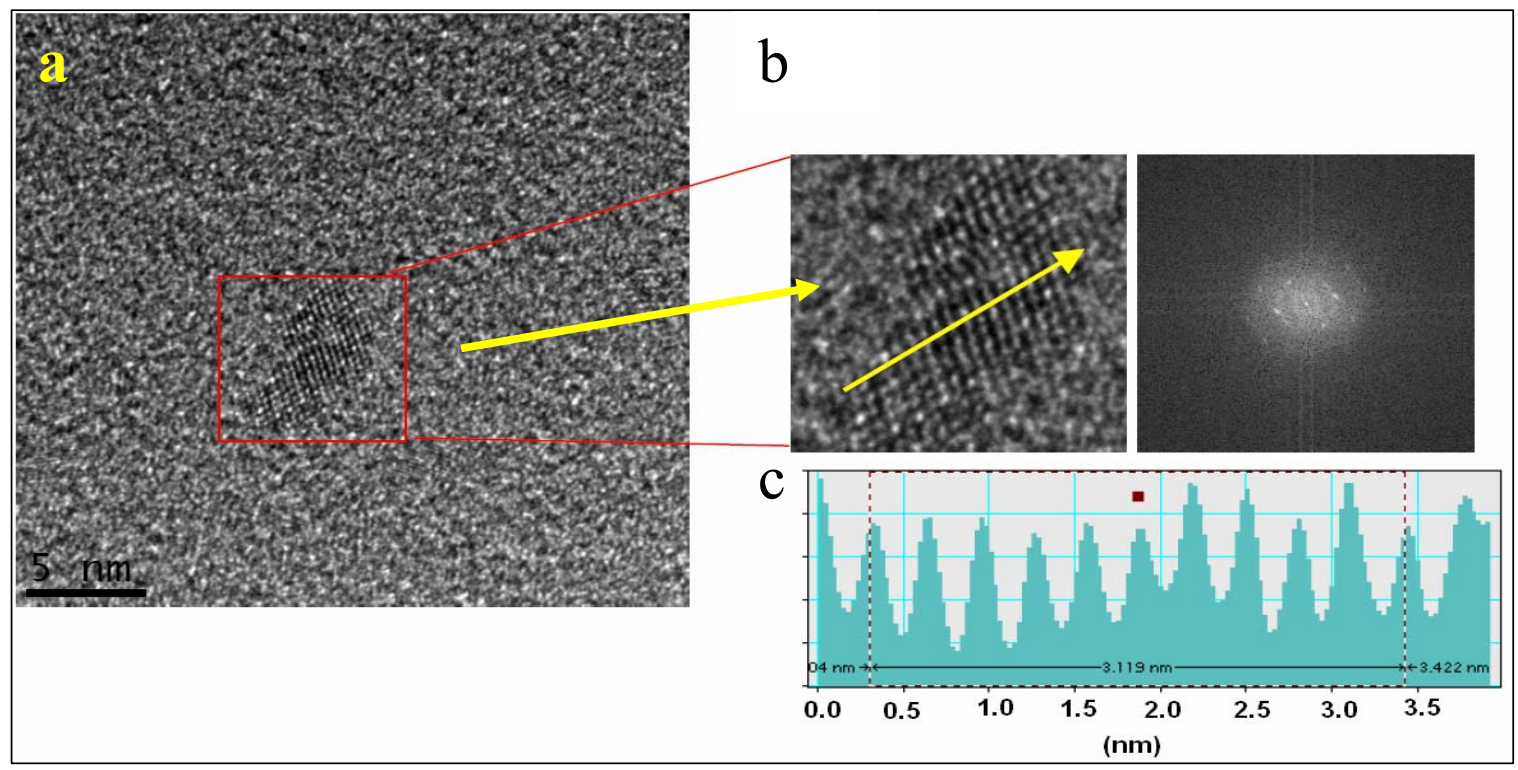

Fig. 2. a) Nanocrystal silicon embedded into matrix amorphous. b) Fourier Transform of nanocrystal. c) Measurements of interplanar distances. 